\title{
MEASUREMENT METHODS OF INTERNAL STRESS IN CONTINUOUS WELDED RAIL
}

\author{
Petr Vnenk*, Bohumil Culek
}

\author{
Department of Transport Structures, Faculty of Transport Engineering, University of Pardubice, Studentska 95, \\ Pardubice, Czech Republic \\ * corresponding author: petr.vnenk@student.upce.cz
}

\begin{abstract}
This paper deals with the problem of internal rail stress estimation. It is based on a detailed research of contemporary situation in the field, presents basic outlines of the problem and sums up the major research areas and their possible applications in the current state of railway infrastructure management.

The directions are divided into four categories in the paper: Displacement Methods, Rail Shifting Methods, Methods Based on Acoustoelastic Effect and Methods Based on Magnetoelastic Effect. Particular methods, both scientific and industrial, are presented in their section respectively.

Every method that is presented within the scope of this paper is briefly described and its advantages and disadvantages are mentioned. In the end, potential of application of some of the presented methods in the practical use is discussed.
\end{abstract}

KEYWORDS: rail, stress, measurement, CWR, temperature.

\section{INTRODUCTION}

Continuous welded rail, abbreviated as CWR, is a part of railway superstructure that has been under continuous development since its beginnings in the first half of the twentieth century. The implementation of continuous welded rail caused a major qualitative improvement in the railway transport. One of the biggest challenges of this improvement was the mitigation of the thermal stress effect.

At the moment of welding, there is a certain stress state which equals a temperature called "neutral temperature". The neutral temperature is characterized by the attribute that when a welded rail reaches the neutral temperature, the rail is free from thermal stress. When the rail temperature is higher than the neutral temperature, compressive stress in rail emerges. At extreme temperatures, the compressive stress induced in the rail can cause railway track buckling, also known as "sun kink". On the contrary, when the rail temperature is lower than the neutral temperature, tensile stress in rail emerges. At extreme temperatures, the tensile stress induced in the rail can cause the rail to be pulled apart.

The thermal stress in the continuous welded rail is expressed as

$$
\sigma=-\alpha \cdot \Delta t \cdot E
$$

where

$$
\alpha=12 \cdot 10^{-6} K^{-1}
$$

and

$$
E=210 \cdot 10^{9} \mathrm{~Pa}
$$

In the Czech Republic, the permitted welding temperature for rails ranges from $17^{\circ} \mathrm{C}$ to $23^{\circ} \mathrm{C}[1$. Considering the minimum rail temperature at $-30^{\circ} \mathrm{C}$ and the maximum rail temperature at $60^{\circ} \mathrm{C}$ (for rails situated in the sunny locations and thus heated), the difference between the extreme temperature and the neutral temperature can reach as many as $53^{\circ} \mathrm{C}[2$.

Assuming such conditions, the thermal stress in continuous welded rail is

$$
\sigma=133.56 \cdot 10^{6} \mathrm{~Pa}
$$

This stress as such is not high enough to cause failure of continuous welded rail. However, there are other contributing factors, too. The thermal stress is cyclic, repeating in both daily and yearly cycles. Trains cause further heavy dynamic loading which is cyclic, too. All these loadings are countable.

It appears, however, that the neutral temperature is not constant over a period of time. Based on the location of the rail, it seems the neutral temperature is shifting. This shift of the neutral temperature can reach even several degrees of Celsius. This difference has to be added to the $\Delta t$ parameter, eventually increasing the thermal rail stress.

Combinations of the above mentioned stresses and their cyclic character have adverse influence on continuous welded rails. Unlike the other items, it is very difficult to predict the shift of the neutral temperature. This article presents contemporary findings in 
this area and suggests possible solutions to the evaluation of the thermal stress in rail with the influence of the neutral temperature shift.

\section{Displacement Methods}

Displacement methods of CWR stress measurement work on the simple mechanical assumption of change of a relative distance between two points of material, rail in this case. In Newton's mechanics, relatively stiff rail behaves according to Hook's law if unfastened. However, even the fastening systems ensure only a certain level of rigidity and, provided the axial force in rail is strong enough, a relative displacement can be observed even at fastened rail sections. As of now, some solutions using displacement are available as presented below.

\subsection{Research of the Gdansk University of TeChNOLOGY}

This research was carried out in the mid-seventies at the Gdansk University of Technology, Poland. A displacement sensor with accuracy of $0.001 \mathrm{~mm}$ has been applied for a measurement on a $200 \mathrm{~mm}$ long rail section. Next to the CWR measurement spot, a reference rail section has been placed. This reference rail section has been set free to lengthen and shorten. It was able to observe soon that the stress varies over different sections of the rail even if the temperature of the rail was the same at measured locations.

It is believed that this effect is caused by slip of rail in the fastening points. Such a slip can be derived by several sources like tangential forces of traffic, but also by exposure of some parts of rail to sun and location of some parts of rail to completely shady place. Such a different exposure to sun results in different daily mean temperature and, subsequently, to a stress gradient in the rail.

This revealing is a good asset of the measurement. The method itself, however, is not suitable for commercial use as it is very costly, time demanding and requires a reference rail to be placed at the measurement spot. Nevertheless, it can well serve as a reference method for calibration of other methods 3 .

\subsection{The Calibrated Length of Rail METHOD}

This method, which has supposedly been applied in China since 1982, consists of length comparison of a freely placed $50 \mathrm{~m}$ long steel strip with a selected rail section originally of the same length. The principle of this method, however, appears to be the same like in the research of the Gdansk University of Technology, only modified for a bigger span. Author's daring claim that this method is the only one in the world without "fatal" shortcomings and can be operated widely does not match the state of the research in this area after the publication date, because much of research has been emerging in this field [4].

\subsection{Measurement With Bi-Directional FBG Strain Sensors}

One of the most recent papers covering this topic, from 2015 , presents a method of displacement measurement in a rail web in longitudinal and vertical direction using optic FBG sensors. In general, this measurement resembles strain gauge measurement. However, the advantage of FBG sensors is the possibility to measure even temperature, apart from mere strain. Moreover, it is characterized by its longer durability in relation to strain gauge sensors. Provided the sensor is attached to the rail web prior to CWR installation (i.e. at rail neutral temperature or known rail tension), it keeps measuring the deformation as long as the sensor works. This can be theoretically reached using strain gauges only, although the durability appears as an important factor here. [5].

\subsection{VORTOK Measure AND Detect}

Another option to measure displacement can be reached by using the Measure and Detect sensor produced by VORTOK Ltd. company. Simple sensor that is able to measure variety of parameters is made ready to be screwed into a drilled hole in a rail web. The manufacturer does not share any detailed information of what principles this device uses. It looks, however, like a piezoelectric sensor with accessories that is incorporated into a screw-in dowel.

This robust shape most probably predicts a displacement method with a long durability. A disadvantage of this method is a necessity of calibration. After calibration is made, either by fastening of the device on a stress-free rail or by using another calibration method, the value of internal stress in rail can be obtained in a stable manner. The options to set up automatic data transmission via email or wi-fi even from a moving train make this sensor a user-friendly device [6].

\section{Rail Shifting Methods}

Rail shifting methods of CWR stress measurement use the principle of bending stiffness dependence on axial tension in rail. Provided the rail is in tension, the higher force is necessary to be applied to laterally shift the rail the higher the axial force in the rail is. Apparently, these methods are viable only when the rail is in tension, otherwise failure by buckling may appear.

\subsection{VERSE METHOD}

This method has been presented by VORTOK Ltd. company from the United Kingdom. In the first place, the rail shall be released from all fastenings in the length of $30 \mathrm{~m}$. Afterwards, the central part of the unfastened section of the rail shall be lifted into a certain height. The force that is applied to move the rail is related to the axial force in the rail.

Wide use of this method is restricted by some disadvantages. The most obvious one is the limitation to tensile stress in the measured rail. The operator needs 
to be sure that there is a tensile stress as problems with re-installation of the rail into the original position could occur, leave apart the danger of operator's injury or material damage. Another disadvantage is the need to unfasten the rail in the length of $30 \mathrm{~m}$. This operation requires additional time and subsequently prolongs track closure. Moreover, in case the measured section is situated in radius, re-installation of the rail can be difficult, too, as the condition of tensile stress in rail is inevitable.

Even though the disadvantages are important, this method appears to be one of the most widely spread to measure the CWR stress nowadays. The manufacturer produces their own device for rail lifting and stress measurement. Light aluminium design of this device enables easy transport, manipulation and operation [7].

\subsection{Use of TAmping Machine}

In a paper published in 2009, scientists at Gdansk University of Technology look into a possibility to use tamping machine for stress measurement in CWR. Tamping machine is capable to shift rails in both vertical and horizontal direction when adjusting railway track geometry. The goal of this research was to use this movement to estimate the stress in CWR. The principle of this measurement is similar like at the VERSE method, including the disadvantages. However, the disturbing signal produced by the operation of tamping machine turned out to be too high and the attempts to get some CWR stress data were unsuccessful [8].

\section{Methods Based on Acoustoelastic Effect}

The acoustoelastic effect is a physical effect of change of sound velocity based on change of mechanical stress in elastic continuum. For measurements, ultrasound is used. The definition, however, can cover vibration, as mechanical waves, too. In such a case, vibrometer is used as a measurement tool.

It is always necessary to execute a calibration measurement on the site with known temperature and mechanical stress as the sound velocity in rail is not dependent only on mechanical stress, but on steel microstructure, too. This calibration measurement can be performed either prior to CWR installation, or once the CWR is installed. However, in the latter only when the rail is cut, unfastened and welded again. As a consequence, similar disadvantage like at displacement methods emerges, which means the need to start the measurement at the neutral temperature. The advantage, on the contrary, is in possible high durability of ultrasound sensors and their attachment to measurement place.

\subsection{J. Szelazek Measurement}

J. Szelazek has worked out two ultrasound measurement procedures in the nineties of the twentieth cen- tury. In the first procedure, ultrasound signal is emitted vertically from the top of rail head to the bottom of rail foot where it reflects off and moves back to the receiver, which can be the same device as the emitter. The second procedure uses separate emitter and receiver and those are placed to the side of rail head horizontally next to each other. The signal moves through the rail head lengthwise, in this case.

The first procedure showed as inapplicable, as the signal dispersed due to uneven rail head profile. The major cause of this unevenness is the rail head wear. Placing the signal receiver to the bottom of rail foot did not improved the results and, moreover, the signal path was reduced to one half.

The second procedure appeared more promising, but even at this measurement, disturbances appeared and the results were influenced by strong dispersion 9 .

\subsection{Research of the University of FLORENCE}

In 2007, Italian scientists worked out the second procedure of J. Szelazek. This procedure has been tested for 2 years and appears viable. However, it shares all the common disadvantages of ultrasound methods and the necessity to perform calibration measurement in situ [10].

\subsection{Measurement of Stresses Using the Polarization of Rayleigh Surface WAVES}

Two US researches work with measurement of polarization of Rayleigh surface waves. Measurement of dependence of the Rayleigh surface waves polarization on change of mechanical rail stress turned out not just to be more robust than measurement of dependence of the Rayleigh surface waves velocity on change of mechanical rail stress, but also to be easier to detect.

Michael D. A. Junge's work deals with general measurement of mechanical stress in material based on Rayleigh surface waves 11. Stefan Hurlebaus' report deals with application of this procedure on rail stress research. On page 42 , the author presents that the polarization of Rayleigh surface waves is dependent on the value of axial force in rail and that a further research is recommended [12].

\subsection{Vibration Measurement}

Two publications, a diploma thesis and a research report, deal with vibration measurement of the internal rail stress. Both of them are outputs of one research. The results of the rail stress determination from wave lengths of vibrating sensor seem well in laboratory conditions when a steel bar or a new rail is used. In case of worn rail, and this is very important for practical application, it is possible to get certain results, but these results are not that clear like in the previous cases. When performing a field measurement, the results of measurement on worn rail are much worse. 
As of yet, this method is inapplicable. In case it is possible to diminish the problems, this method can have a good perspective [13, 14.

\section{Methods Based ON MAGNeTOELASTIC EFFECT}

The magnetoelastic effect is inverse magnetostrictive effect. It is presented as a change of magnetic susceptibility in relation to change of mechanical stress in material. The magnetoelastic effect is also known as the Villari effect.

\subsection{Measurement of Magnetic HYSTERESIS}

In the nineties, D. Utrata tested the possibility of application of the magnetoelastic effect on the rail stress measurement. He measured the dependence of coercivity and remanence of the magnetic field in rail on different surface conditions, like scaled rail surface, or milled rail surface. However, the comparison of the obtained data has shown various results based on the input type of rail. Surprisingly, the only data that match were on the milled running surface and scaled base underside. D. Utrata assumes, in the end, that either a calibration measurement or a new approach to data interpretation has to be delivered in order to make this method applicable.

A. Wegner needed 400 calibration sensors to run a measurement of 3 meters long rail using the hysteresis method, which does not contribute to viability of this method [14, 15].

\subsection{Measurement of Magnetic BARKHAUSEN EFFECT}

Japanese scientists Tsuchima and Enokinozo have studied the reaction of the Barkhausen noise on mechanical stress in steel plate. It is possible to get some relation from the graphs of the mutual dependence. However, the authors found it difficult to estimate the influence of time and get a value of the mechanical stress. Additionally, they suggest application of the chaos theory to get a better interpretation of the results [16].

Measurement using the Barkhausen noise together with measurement of magnetic permeability is used by Elektro-Thermit GmbH \& Co. KG. company to determine the internal rail stress. Their method requires calibration on a rail test sample. Their product has been awarded a certificate of the railway infrastructure manager of Denmark, Banedanmark. On the contrary, the manufacturer appears not interested in sale of their product and only offers to perform a measurement in the required section by themselves [3, 17].

\subsection{Metal Magnetic Memory MeAsurement}

Collective of authors from the University of Nanjing have carried out an experiment of measuring the effect called Metal Magnetic Memory on a steel test sample.

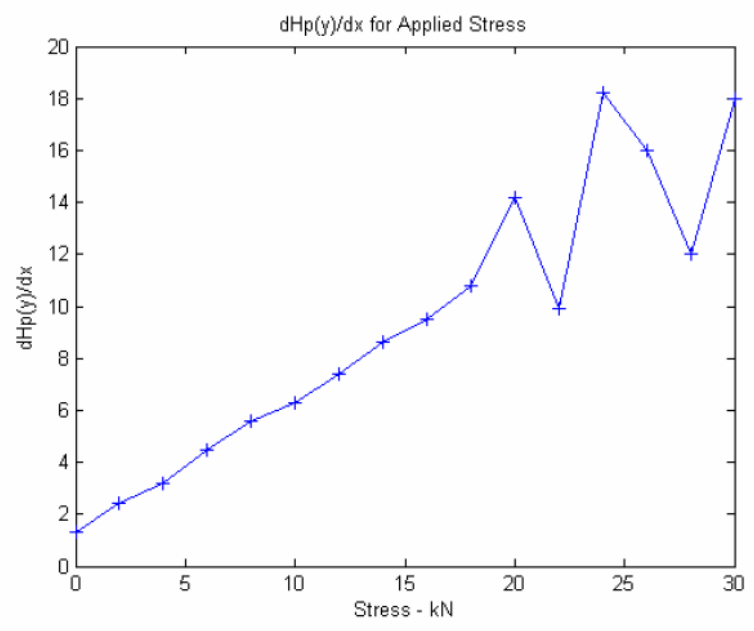

Figure 1. $\mathrm{dHp}(\mathrm{y}) / \mathrm{dx}$ for applied tensile stress on a steel sample [18; see the linear dependence in the elastic region.

Contrary to the Barkhausen noise, this effect does not require external magnetization of the sample. The effect is activated anywhere in the natural magnetic field of the Earth. When the loading increases, the rotation of the Weiss magnetic domains in metals raises the value of the magnetic flux around the central point and this increases the gradient of the magnetic flux which is in elastic region linearly dependent on the stress in the sample. Graph of the linear dependence of the magnetic flux gradient on the mechanical stress in a steel sample as measured by Wang, et. al. [18] is presented in Figure 1. The linear dependence in the elastic region is completely sufficient, because rail geometry deformation emerges earlier than plastic deformation in the axial direction of the rail [18].

\section{Discussion}

Every presented method has its limitation. Displacement methods can be related to widely spread strain gauge measurement. This has been used in many industrial application; in this case, however, the biggest problem seems to be the necessity to measure an absolute value of stress, not a relative one, which is the common purpose of strain gauge measurement in general.

Rail shifting methods, especially the VERSE method, seem to be one of the most reliable at this time. The reliability is underpinned by its simplicity. However, more extensive use of this method is very limited through many disadvantages it inevitably drags along. Movement of a rail is a complicated operation, which needs expensive tools and is very time demanding. Re-installation of the rail requires verification that the rail is in good condition after the measurement and safety of operation on the track is kept. Additionally, performing such a measurement in radius is much more complicated and performing such a measurement when the rail is under a compressive stress is excluded. Considering the compressive stress 
in rail is usually during the day, the limitations of use of this method are vast.

Methods based on the acoustoelastic and magnetoelastic effect have the potential to become viable in the non-destructive rail stress measurements. As of yet, however, the problems of getting clear values of stress were not mitigated and residual stresses, difficult conditions of field measurement, or even various conditions of the rail itself (wear, rust, etc.) prevented the research to reach a satisfactory solution.

\subsection{Further Research Orientation}

Out of the contemporary possibilities, there are some ways to be worked out in order to either get satisfactory results or to discover serious obstacles that hinder their application.

(1.) Application of the Metal Magnetic Memory Method on internal rail stress measurement seem like a research that has not been done yet. As mentioned in Section 5 this method does not require external magnetization 18 and this is a great asset for possible further application. If the measurement of the gradient of magnetic flux in rail results in a similar dependence as shown in Figure 11 the potential of applicability of this method would be great [18.

(2.) Vibration measurement, or modal analysis could also offer an approach to obtain data of internal stress in CWR. However, damping caused by rail fastenings could have too high influence on the results. As presented in Section 4 , the vibration analysis performed by the team of the University of Illinois at Urbana-Champaign did not produce any applicable results even after they released some fastenings, which is a procedure that is disadvantageous as such [13, 14].

(3.) Provided the stress could be evaluated from a surface layer of rail only, i. e. there was a mutual relation between surface stress and stress in the core of the rail, another possible method emerges. Method of dependence between passive layers formation in electrolyte and mechanical stress in the surface layers has been shown in an experiment of stainless steel in a normal sulphuric acid bath [19].

(4.) Another option is to work out the measurement of stresses using the polarization of Rayleigh surface waves. The papers presented in Section 4 suggest a further research can have a potential to find out a viable solution [11, 12.

(5.) Finally, if strain gauge is able to measure a quasi static load change in a long term, a statistical evaluation of measurements could provide a range of data that could be sufficient for prediction of neutral temperature level and subsequently stress level in rail. If such a research is biased towards a certain limited location, an applicable methodology can be a reasonable output.

\section{Conclusions}

Many attempts to measure the neutral temperature or stress in continuous welded rail have been undertaken. Displacement and rail shifting methods like VORTOK Measure and Detect or VERSE have been finalized in an applicable product and offered on market. These methods come out of mechanical solutions. However, these methods suffer from many limitations and requirements of special conditions that are necessary to be created in order to use the methods.

Later in time, non-destructive methods based on the acoustoelastic or magnetoelastic effects have been tested. As of yet, none of them reached the level of being reliable enough to compete with the above mentioned ones. Nevertheless, there are some promising results and further research in this area, as described in Section 6, could prove viability of some of them.

Provided strain gauges are capable of measuring quasi static load in a long term and a thorough strain gauge measurement is done, the value of stress in a continuous welded rail could be estimated on a certain geographical area even from statistical data measured by strain gauges.

Finally, the possible solution can lurk in another method of stress evaluation in a continuum, a method which is not yet known to the authors of this paper.

\section{ACKNOWLEDGEMENTS}

The work was created in connection with the scientific research project of the University of Pardubice No. SGS_2017_009. The authors are grateful for the support.

\section{REFERENCES}

[1] Sprava zeleznicni dopravni cesty, s.o. SZDC S 3/2 Bezstykova kolej, 2013. Kapitola III, Cast A, Bod 110.

[2] Sprava zeleznicni dopravni cesty, s.o. SZDC S 3 dil IV Zeleznicni svrsek. Kolejnice ve zneni zmen c. 1 a 2, 2014. Kapitola IV, Bod 43.

[3] W. Koc, et. al. Okreslanie wartosci sil podluznych w szynach toru bezstykowego. Problemy Kolejnictwa 163, 2014.

[4] L. Xing-han. The "Calibrated length of rail" method for measuring rail temperature stress. AREMA Proceedings of the 2000 Annual Conference 2000.

[5] P. Wang, et. al. Longitudinal force measurement in continuous welded rail with bi-directional FBG strain sensors. Smart Materials and Structures 25, 2015.

[6] Vortok, Ltd. Vortok measure and detect, 2012. Vortok, [2016-06-07], http://www.vortok.com/rail-stress-management/ vortok-measure-and-detect-2

[7] Vortok, Ltd. VERSE, 2012. Vortok, [2016-05-31], http: //www.vortok.com/rail-stress-management/verse

[8] W. Koc, A. Wilk. Investigations of methods to measure longitudinal forces in continuous welded rail tracks using the tamping machine. Journal of Rail and Rapid Transit 223(1), 2009. 
[9] J. Szelazek. Monitoring of thermal stresses in continuously welded rails with ultrasonic technique, 1998. NDTnet, 1. June 1998, [2016-05-30], http://www. ndt.net/article/dresd97/szelazek/szelazek.htm

[10] D. Vangi, A. Virga. A practical application of ultrasonic thermal stress monitoring in continuous welded rail. Experimental Mechanics 47, 2007.

[11] M. D. A. Junge. Measurement of applied stresses using the polarization of rayleigh surface waves, 2003. Atlanta, Georgia, School of Civil and Environmental Engineering, Institute of Technology.

[12] S. Hurlebaus. Determination of longitudinal stress in rails, 2011. College Station, Texas, Transportation Research Board of the National Academies.

[13] R. Weaver. Vibration measurement of rail stress, 2006. Champaign, Illinois, Transportation Research Board of the National Academies.

[14] M. J. Koob. The development of a vibration technique for estimation of neutral temperature in continuously welded railroad rail, 2005. Champaign, Illinois, University of Illinois at Urbana-Champaign.
[15] D. Utrata, A. Strom, M. Negley. Stress measurement in railroad rail using ultrasonic and magnetic technique. Review of Progress in Quantitative Nondestructive Evaluation 14, 1995.

[16] Y. Tsuchida, M. Enokinozo. Stress evaluation by chaotic characteristics of barkhausen noise. Review of Quantitative Nondestructive Evaluation 22, 2003.

[17] A. Wegner. Stress-free temperature monitoring using different measuring technologies - experiences and assessment. Proceedings of 10th International Heavy Haul Association Conference 2013.

[18] P. Wang, et. al. Stress measurement using magnetic barkhausen noise and metal magnetic memory testing. 17th World Conference on Nondestructive Testing 2008.

[19] F. Navai. Effects of tensile and compressive stresses on the passive layers formed on a type 302 stainless steel in a normal sulphuric acid bath. Journal of Materials Science 30, 1995. 